\title{
Candidate gene association study in pediatric acute lymphoblastic leukemia evaluated by Bayesian network based Bayesian multilevel analysis of relevance
}

Orsolya Lautner-Csorba ${ }^{1}$, András Gézsi ${ }^{1}$, Ágnes F Semsei ${ }^{1}$, Péter Antal ${ }^{2}$, Dániel J Erdélyi ${ }^{3}$, Géza Schermann ${ }^{1}$, Nóra Kutszegi ${ }^{1}$, Katalin Csordás ${ }^{3}$, Márta Hegyi ${ }^{3}$, Gábor Kovács ${ }^{3}$, András Falus ${ }^{1}$ and Csaba Szalai ${ }^{1,4,5^{*}}$

\begin{abstract}
Background: We carried out a candidate gene association study in pediatric acute lymphoblastic leukemia (ALL) to identify possible genetic risk factors in a Hungarian population.

Methods: The results were evaluated with traditional statistical methods and with our newly developed Bayesian network based Bayesian multilevel analysis of relevance (BN-BMLA) method. We collected genomic DNA and clinical data from 543 children, who underwent chemotherapy due to ALL, and 529 healthy controls. Altogether 66 single nucleotide polymorphisms (SNPs) in 19 candidate genes were genotyped.
\end{abstract}

Results: With logistic regression, we identified 6 SNPs in the ARID5B and IKZF1 genes associated with increased risk to B-cell ALL, and two SNPs in the STAT3 gene, which decreased the risk to hyperdiploid ALL. Because the associated SNPs were in linkage in each gene, these associations corresponded to one signal per gene. The odds ratio (OR) associated with the tag SNPs were: $\mathrm{OR}=1.69, \mathrm{P}=2.22 \times 10^{-7}$ for $\mathrm{rs} 4132601$ (IKZF1), $\mathrm{OR}=1.53, \mathrm{P}=1.95 \times 10^{-5}$ for rs 10821936 (ARID5B) and OR=0.64, $\mathrm{P}=2.32 \times 10^{-4}$ for rs 12949918 (STAT3). With the BN-BMLA we confirmed the findings of the frequentist-based method and received additional information about the nature of the relations between the SNPs and the disease. E.g. the rs10821936 in ARID5B and rs17405722 in STAT3 showed a weak interaction, and in case of T-cell lineage sample group, the gender showed a weak interaction with three SNPs in three genes. In the hyperdiploid patient group the BN-BMLA detected a strong interaction among SNPs in the NOTCH1, STAT1, STAT3 and BCL2 genes. Evaluating the survival rate of the patients with ALL, the BN-BMLA showed that besides risk groups and subtypes, genetic variations in the BAX and CEBPA genes might also influence the probability of survival of the patients.

Conclusions: In the present study we confirmed the roles of genetic variations in ARID5B and IKZF1 in the susceptibility to B-cell ALL. With the newly developed BN-BMLA method several gene-gene, gene-phenotype and phenotype-phenotype connections were revealed. We showed several advantageous features of the new method, and suggested that in gene association studies the BN-BMLA might be a useful supplementary to the traditional frequentist-based statistical method.

Keywords: ALL susceptibility, Bayesian network based Bayesian multilevel analysis of relevance (BN-BMLA), Frequentist-based statistical analysis, Gene-gene interaction, Genetics, Genomics, Risk factors, Direct and indirect interactions, Transitive interaction, Strong relevance, Systems biology

\footnotetext{
* Correspondence: genomika.cs@gmail.com

'Department of Genetics, Cell- and Immunobiology, Semmelweis University, Budapest, Nagyvárad tér $4 \mathrm{H}-1089$, Hungary

${ }^{4}$ Heim Pal Children Hospital, Budapest, Hungary

Full list of author information is available at the end of the article
} 


\section{Background}

Pediatric acute lymphoblastic leukemia (ALL) is a clonal disease of a lymphoblast and the most common malignancy of all childhood cancers. It is generally accepted that tumorogenesis results from complex interplay between inherited genetic background and specific environmental exposure [1]. In the last decade several genome wide and candidate gene association studies have been carried out and revealed a number of genes and genetic variations, which might influence the risk to the disease [2-6]. In gene association studies, due to the high number of inconclusive results, it is generally accepted that the role of a gene or a genetic variation can only be acknowledged, if it is confirmed by independent studies.

In gene association studies it is well-known, that the traditional frequentist-based statistical methods have several limitations, like the difficult handling of the multiple testing problem and model complexity, the inappropriate approach towards complex traits (i.e. disregarding the role of high-number of weak factors, genegene interactions, pathway-based interpretation), and the high redundancy of predictors, e.g. the discovery of non-causal, transitively associated descriptors $[7,8]$.

Recently, we have introduced a new statistical methodology, named Bayesian network based Bayesian multilevel analysis of relevance (BN-BMLA), which supports association analysis by estimating posteriors of strong relevance [9-11]. First, we tested the BN-BMLA method in a case-control setup using artificial datasets for identifying interactions and conditional relevance [12]. The BN-BMLA was proven to be superior over other multivariate methods using conditional models designed to detect associations between genotypic variables and the target variable. Later, we also tested the method in a real world dataset of a partial genome association study in asthma [13]. In this latter study we found that next to the directly associated genes identified by the frequentist-based methods ( $\chi^{2}$ test, multivariate logistic regression and multifactor dimensionality reduction), the BN-BMLA could detect plausible additional genes involved in gene-gene interactions or genes which were indirectly associated with asthma, i.e. showed transitive association via other variables (e.g. via rhinitis).

As could be seen from the results, the advantage was not only that the BN-BMLA could detect more relevant variables, but the Bayesian networks offered a rich language for the detailed representation of types of relevance, including causal, acausal, and multitarget aspects. Additionally, Bayesian statistics offers an automated and normative solution for the multiple hypothesis testing problems. The BN-BMLA extends the scope of local 'causal' discovery methods, and because of the direct interpretation of Bayesian posteriors, contrary to $\mathrm{p}$-values from the frequentist-based approach, makes it an ideal candidate for creating probabilistic knowledge bases to support off-line meta-analysis and fusion of background knowledge.

In the present study we use a traditional frequentistbased method (logistic regression) and the BN-BMLA for evaluating the results of a candidate gene association study in ALL. From the scientific literature and databases we selected 66 SNPs in 19 known candidate genes and investigated with the two methods whether the presence of these polymorphisms was associated with ALL in our population, and whether any of these alleles influenced some disease characteristics and the outcome of the therapy in the affected individuals.

\section{Methods}

\section{Study population and definitions}

In a retrospective manner, DNA was obtained from 543 children (mean age at diagnosis: $6.4 \pm 4.2$ years) who underwent chemotherapy due to acute lymphoblastic leukemia. Patients were diagnosed with ALL between 1990 and 2010, aged 1-15 years at diagnosis and treated according to the ALL Berlin-Frankfurt-Münster (BFM) 90, 95 and 2002 chemotherapy protocols in ten Hungarian centres.

We stratified our patients in different risk groups according to the following criteria: Low risk (LR) group included children aged 1-6 years who have a white blood cell count of less than $20,000 / \mu \mathrm{l}$ at diagnosis, good prednisone response and no-T-ALL. High risk (HR) group included poor prednisone response, and/or evidence of $\mathrm{t}(9 ; 22)$ (or BCR/ABL), and/or evidence of $\mathrm{t}(4 ; 11)$ (or MLL/AF4). Medium risk (MR) group included children with no HR or LR criteria. Prednisone responses were determined after 8 and 33 days of induction treatment. The presence of $1,000 \mathrm{blast} / \mu \mathrm{l}$ or more in the peripheral blood on day 8 and/or $>5 \%$ blast in the bone marrow on day 33 were defined as poor prednisone response.

There is no significant difference in the distribution of age groups or genders or ALL-immunophenotypes between the whole population and our sample-collection.

The 529 control patients (aged 16.1 \pm 12.4 years) of the same ethnicity and from the same geographical region as the patients were randomly selected from healthy blood donors and from minor outpatients from the Orthopaedic Department in the Budai Children's Hospital, and from the Urological Department of Heim Pal Pediatric Hospital, Budapest. None of the controls have had childhood ALL or any other types of cancers previously. In Table 1, some clinical characteristics of ALL patients and controls are presented.

All study subjects belonged to the Hungarian (Caucasian) population. Informed consent was requested from 
Table 1 Some characteristics of ALL patients and controls in the study population

\begin{tabular}{|c|c|c|c|}
\hline Characteristics & Subgroups & ALL & Controls \\
\hline Total number of subjects & & 543 & 529 \\
\hline \multirow[t]{2}{*}{ Gender n (\%) } & Male & $308(56.7)$ & $305(57.7)$ \\
\hline & Female & $235(43.3)$ & $224(42.3)$ \\
\hline $\begin{array}{l}\text { Age at diagnosis, } \\
\text { years (mean } \pm S D)\end{array}$ & & $6.4 \pm 4.2$ & $16.1 \pm 12.4$ \\
\hline \multirow[t]{4}{*}{ Number of subjects n (\%) } & $<1$ year & $8(1.5)$ & - \\
\hline & 1-10 years & $422(77.7)$ & $200(37.8)$ \\
\hline & $>10$ years & $107(19.7)$ & $329(62.2)$ \\
\hline & $\mathrm{N} / \mathrm{D}$ & $6(1.1)$ & - \\
\hline \multirow[t]{3}{*}{ Risk group n (\%) } & LR & $96(17.7)$ & - \\
\hline & $M R$ & $309(56.9)$ & - \\
\hline & $H R$ & $55(10.1)$ & - \\
\hline \multirow[t]{4}{*}{ Protocols n (\%) } & 90 & $131(24.1)$ & - \\
\hline & 95 & $250(46.0)$ & \\
\hline & 2002 & $120(22.1)$ & - \\
\hline & N/D & $42(7.7)$ & \\
\hline \multirow[t]{2}{*}{ Immunphenotype n (\%) } & $B-A L L$ & $390(71.8)$ & - \\
\hline & $\mathrm{T}-\mathrm{ALL}$ & $78(14.4)$ & - \\
\hline Cytogenetics n (\%) & Hyperdiploidy & $79(14.5)$ & - \\
\hline Overall survival n (\%) & $516^{*}$ & $414(85.5)^{*}$ & - \\
\hline Event free survival n (\%) & $516^{*}$ & $418(81.0)^{*}$ & - \\
\hline
\end{tabular}

N/D: no data available; LR: Low risk, MR: Medium risk; HR: High risk, *see details in the text.

the study subjects, or from the parents of patients. The study was conducted according to the principles expressed in the Declaration of Helsinki and was approved by the Hungarian Scientific and Research Ethics Committee of the Medical Research Council (ETT TUKEB; Case No.:8-374/2009-1018EKU 914/PI/08.)

\section{Candidate gene selection}

From the scientific literature we selected 19 candidate genes, which in earlier studies were seemed to be relevant to ALL. We selected genes from the results of GWA studies, candidate gene association studies and from other studies in which the investigated pathways could also be important for ALL. In online databases we searched SNPs for these 19 genes. Altogether 66 SNPs were selected. The selection criterion was: minor allele frequency $>10 \%$. The SNPs were prioritized according to their published role in ALL, and their estimated functionality. In some cases (e.g. in ARIDB5, IKZF1 genes) SNPs were selected, which showed strong linkage disequilibrium with other investigated SNPs in the HapMap database [14]. This could serve as genotyping controls, but during the BN-BMLA evaluation tag SNPs were chosen for the analysis. Table 2 shows information about the selected genes and SNPs.

\section{Laboratory methods, genotyping}

Genomic DNA from children with leukemia was obtained retrospectively from whole, peripheral blood taken in remission phase using by QIAmp DNA Blood Maxi Kit (Qiagen, Hilden, Germany) according to the manufacturer's instructions. Before DNA extraction the normal leukocyte cell count was checked by FACS. We used only those samples where the normal cell count was over $5 \mathrm{G} / \mathrm{l}$. DNA from healthy control samples was isolated from whole peripheral blood using iPrep PureLink gDNA Blood Kit, iPrep Purification Instrument (Invitrogen, Life Technologies Co., Grand Island, USA). Genotyping was carried out by Sequenom iPLEX Gold MassARRAY technology at the McGill University and Génome Québec Innovation Centre, Montréal, Canada. Only those SNPs were included in the analysis, which have a genotyping call rate over $90 \%$.

\section{Frequentist-based statistical analysis}

Allele frequencies were calculated by allele counting. Hardy-Weinberg equilibrium was tested by using a $x^{2}$ goodness-of-fit test with an acceptable cut off value of $\mathrm{p} \geq 0.01$ (online application: [15]).

The distributions of alleles and genotypes in cases and controls were compared by means of the $x^{2}$ test [15]. Association between the candidate SNPs and the susceptibility to childhood acute lymphoblastic leukemia was tested using gender adjusted logistic regression in the case-control data performed by IBM SPSS Statistics software (version 19.0). Confidence interval was calculated at the 95\% level (C.I. 95\%). Multiple testing corrections were performed using the Benjamini-Hochberg false discovery rate (FDR) method with type I error rate of $1 \%$.

Haplotype analyses were carried out by Haploview version 4.2 software (Broad Institute, Cambridge, Maine, USA). Odds ratios for haplotypes were calculated by MedCalc 12.1.1 software (MedCalc Software, Mariakerke, Belgium). Survival analyses were performed using Kaplan-Meier method. The log-rank test was applied for evaluating the association between survival and categorized parameters, as risk group, gender, and studyprotocols. The statistical tests were carried out by IBM SPSS Statistics software, version 19.0.

\section{Bayesian Network based Bayesian Multilevel Analysis}

A Bayesian Network (BN) is a probabilistic graphical model that represents the conditional dependencies of a set of random variables with a directed acyclic graph (DAG). A BN can efficiently describe the joint probability distribution of the variables. A node of the graph 
Table 2 Some information about the selected genes and SNPs

\begin{tabular}{|c|c|c|c|c|}
\hline SNP (rs\#) & Gene & Alleles $(1 / 2)^{a}$ & Position $^{\text {b }}$ & Function $^{c}$ \\
\hline rs2066853 & $A h R$ & $\mathrm{G} / \mathrm{A}$ & chr7:17345635 & missense/Arg $>$ Lys \\
\hline rs713150* & $A h R$ & $C / G$ & chr7:17306682 & intron \\
\hline rs2282885 & $A h R$ & $\mathrm{~T} / \mathrm{C}$ & chr7:17312139 & intron \\
\hline rs2282883 & $A h R$ & $\mathrm{G} / \mathrm{A}$ & chr7:17322872 & intron \\
\hline rs2237297 & $A h R$ & $C / T$ & chr7:17326119 & intron \\
\hline rs10994982* & $A R I D 5 B$ & $A / G$ & chr10:63380110 & intron \\
\hline rs10821936 & $A R I D 5 B$ & $\mathrm{~T} / \mathrm{C}$ & chr10:63393583 & intron \\
\hline rs7089424 & ARID5B & $T / G$ & chr10:63422165 & intron \\
\hline rs4948502 & ARID5B & $\mathrm{T} / \mathrm{C}$ & chr10:63509423 & intron \\
\hline rs4948496 & $A R I D 5 B$ & $\mathrm{~T} / \mathrm{C}$ & chr10:63475623 & intron \\
\hline rs4948487 & $A R I D 5 B$ & $\mathrm{~A} / \mathrm{C}$ & chr10:63339871 & intron \\
\hline rs4506592 & ARID5B & $\mathrm{G} / \mathrm{A}$ & chr10:63397193 & intron \\
\hline rs4509706 & $A R I D 5 B$ & $\mathrm{~T} / \mathrm{C}$ & chr10:63331346 & near-gene-5 \\
\hline rs3817074 & $B A X$ & $C / T$ & chr19:54151024 & intron \\
\hline rs7259013 & $B A X$ & $\mathrm{~A} / \mathrm{C}$ & chr19:54155096 & intron \\
\hline rs11667351 & $B A X$ & $\mathrm{~T} / \mathrm{G}$ & chr19:54147966 & near-gene-5 \\
\hline rs12457893 & $B C L 2 A$ & $\mathrm{~A} / \mathrm{C}$ & chr18:59077141 & intron \\
\hline rs11876772 & $B C L 2 A$ & $A / G$ & chr18:58968686 & intron \\
\hline rs2850761 & $B C L 2 A$ & $A / G$ & chr18:59117377 & intron \\
\hline rs8092560 & $B C L 2 A$ & T/A & chr18:58954673 & intron \\
\hline rs4987845 & $B C L 2 A$ & $\mathrm{G} / \mathrm{A}$ & chr18:58946168 & untranslated-3 \\
\hline rs1801018 & $B C L 2 B$ & $A / G$ & chr18:59136859 & coding-synon/Thr>Thr \\
\hline rs1893806 & $B C L 2 B$ & $T / G$ & chr18:59135944 & near-gene-3 \\
\hline rs1799988 & CCR5 & $C / T$ & chr3:46387263 & untranslated-5 \\
\hline rs3087253 & CCR5 & $\mathrm{T} / \mathrm{C}$ & chr3:46393693 & near-gene-3 \\
\hline rs11575815 & CCR5 & T/A & chr3:46395174 & unknown \\
\hline rs10403561 & CEBPA & $A / G$ & chr19:38482342 & near-gene-3 \\
\hline rs874966 & CEBPA & $A / G$ & chr19:38481237 & near-gene-3 \\
\hline rs2239633 & CEBPE & $\mathrm{C} / \mathrm{T}$ & chr14:22658897 & near-gene-5 \\
\hline rs8015478 & CEBPE & $C / A$ & chr14:22655858 & near-gene-3 \\
\hline rs12434881 & CEBPE & $\mathrm{G} / \mathrm{A}$ & chr14:22658482 & near-gene-5 \\
\hline rs11978267 & $|K Z F|$ & $A / G$ & chr7:50433798 & intron \\
\hline rs4132601 & $|K Z F|$ & $T / G$ & chr7:50438098 & untranslated-3 \\
\hline rs6954833 & $|K Z F|$ & $A / G$ & chr7:50425940 & intron \\
\hline rs10235796 & $|K Z F|$ & $C / T$ & chr7:50430131 & intron \\
\hline rs6964969 & $|K Z F|$ & $A / G$ & chr7:50440745 & near-gene-3 \\
\hline rs11208538** & JAK1 & $\mathrm{G} / \mathrm{C}$ & chr1:65161877 & intron \\
\hline rs310225 & JAK1 & $\mathrm{G} / \mathrm{A}$ & chr1:65097271 & intron \\
\hline rs12063205 & JAK1 & $A / G$ & chr1:65144640 & intron \\
\hline rs3212713 & JAK3 & $\mathrm{G} / \mathrm{A}$ & chr19:17816001 & intron \\
\hline rs11888 & JAK3 & $\mathrm{T} / \mathrm{C}$ & chr19:17796626 & near-gene-3 \\
\hline rs2229974 & NOTCHI & $\mathrm{T} / \mathrm{C}$ & chr9:138511457 & coding-synon/Asp>Asp \\
\hline
\end{tabular}


Table 2 Some information about the selected genes and SNPs (Continued)

\begin{tabular}{|c|c|c|c|c|}
\hline rs3124596 & NOTCHI & $A / G$ & chr9:138521325 & intron \\
\hline rs3124999 & NOTCH1 & $\mathrm{C} / \mathrm{T}$ & chr9:138515294 & intron \\
\hline rs3124603 & NOTCH1 & $\mathrm{T} / \mathrm{C}$ & chr9:138529998 & intron \\
\hline rs1800566 & NQO1 & $\mathrm{C} / \mathrm{T}$ & chr16:68302646 & missense/Pro>Ser \\
\hline rs1469908 & NQO1 & $A / G$ & chr16:68321913 & unknown \\
\hline rs1143684 & NQO2 & $\mathrm{T} / \mathrm{C}$ & chr6:2955389 & missense/Leu>Phe \\
\hline rs2756075 & NQO2 & $\mathrm{C} / \mathrm{T}$ & chr6:2949532 & intron \\
\hline rs4149352 & NQO2 & $\mathrm{C} / \mathrm{T}$ & chr6:2947237 & intron \\
\hline rs2070999 & NQO2 & $\mathrm{G} / \mathrm{A}$ & chr6:2944728 & near-gene-5 \\
\hline rs4149360* & NQO2 & $\mathrm{G} / \mathrm{A}$ & chr6:2951806 & intron \\
\hline rs2030171 & STAT1 & $\mathrm{G} / \mathrm{A}$ & chr2:191577408 & intron \\
\hline rs10208033 & STAT1 & $\mathrm{T} / \mathrm{C}$ & chr2:191587662 & near-gene-5 \\
\hline rs3088307 & STAT1 & $\mathrm{G} / \mathrm{C}$ & chr2:191537657 & untranslated-3 \\
\hline rs12949918 & STAT3 & $\mathrm{T} / \mathrm{C}$ & chr17:37779799 & intron \\
\hline rs3809758 & STAT3 & $\mathrm{G} / \mathrm{A}$ & chr17:37725506 & intron \\
\hline rs3816769 & STAT3 & $\mathrm{T} / \mathrm{C}$ & chr17:37751799 & intron \\
\hline rs17405722 & STAT3 & $\mathrm{G} / \mathrm{A}$ & chr17:37796027 & near-gene-5 \\
\hline rs7217728 & STAT5A & $\mathrm{T} / \mathrm{C}$ & chr17:37700927 & intron \\
\hline rs3198502 & STAT5A & $\mathrm{G} / \mathrm{T}$ & chr17:37716520 & untranslated-3 \\
\hline rs9906933 & STAT5B & $\mathrm{G} / \mathrm{A}$ & chr17:37663571 & intron \\
\hline rs4029774 & STAT5B & $\mathrm{A} / \mathrm{G}$ & chr17:37682487 & near-gene-5 \\
\hline rs703817 & STAT6 & $\mathrm{G} / \mathrm{A}$ & chr12:55776095 & untranslated-3 \\
\hline rs3024979 & STAT6 & $\mathrm{T} / \mathrm{A}$ & chr12:55774560 & intron \\
\hline rs324015 & STAT6 & $G / A$ & chr12:55776367 & untranslated-3 \\
\hline
\end{tabular}

*Failed genotyping, ${ }^{* *}$ Monomorphic, ${ }^{a}$ Alleles on the forward strand; 1 - major allele; 2-risk allele, ${ }^{b}$ Position according to NCBI Genome Build 36.0 , ${ }^{\mathrm{C}}$ Function according to CHIP Bioinformatics Tools http://snpper.chip.org/.

represents a variable and an edge represents a direct dependency between the corresponding variables.

Structure learning is finding a DAG that best describes the dataset. However, in most cases, where the amount of available data is modest relative to the number of variables, usually there are many DAGs that have non-negligible a posteriori probabilities. Even in these cases, there might be certain structural features, e.g. the presence of an edge, which we can extract reliably. The Bayesian learning framework enables us to estimate a posteriori probability of a certain feature $f$ as follows

$$
P(f \mid D)=\sum_{G} f(G) P(G \mid D)
$$

where $G$ represents a $B N$ structure, $D$ is the dataset, and $f(G)$ is an indicator function of the presence of $f$ in $G$, i.e. it is 1 if the feature holds in $G$ and 0 otherwise.

We use Bayes' theorem to calculate $P(G \mid D)$, and we have that $P(G \mid D) \propto P(D \mid G) P(G)$. Assuming that there are no missing values in the dataset $D$, the variables are multinomial with a Dirichlet parameter prior for every possible instantiations of their parents, and the prior $P(G)$ satisfies some general constraints, then the term $P(D \mid G)$ (i.e. the marginal likelihood of the data) can be computed efficiently in a closed form [16]. The other term, $P(G)$ is the a priori probability of a structure $G$. We use uniform prior over structures in our experiments and the $\mathrm{K} 2$ hyperparameter prior, as proposed by Cooper and Herskovits [16].

Because the number of $\mathrm{BN}$ structures is superexponential in the number of nodes, exact summation of all possible structures $G$ is computationally intractable. We use Metropolis-coupled Markov Chain Monte Carlo $\left(\mathrm{MC}^{3}\right)$ methods [17] over the space of DAGs for the approximation of Eq.1. We run the Metropolis-coupled Markov Chain sampler with a burn-in period of $2 \times 10^{7}$ steps and then collect $6 \times 10^{7}$ samples (i.e. network structures). We restrict the space of the possible structures limiting the number of parents per node to 4 . To ensure the convergence of the posterior of the structural features estimated by the MCMC, we use Gelman-Rubin R-scores [18] and Geweke Z-scores [19]. All reported 
posteriors had an R-score below 1.05, and Geweke Zscores also confirmed convergence.

We computed a posteriori probabilities for structural features summarized in Table 3, and as an illustration see Figure 1.

The current implementation of our method deals with only discrete variables. Continuous covariates have to be discretized first in order to include them in the BN-BMLA analysis. In this paper, we use only discrete variables (SNPs, gender, case/control status, 5-year survival).

\section{Computing redundancies and interactions}

In a post-processing step, we can compute the a posteriori probability of the strong relevance of any set of variables with a given size $k[9,10]$. The a posteriori probability of the sub-relevance of a $k$-sized set $s$ is denoted with a special notation as follows $p(s \mid D)=$ $P(M B S(Y)=s \mid D)+\sum_{s \subset s^{\prime}} P\left(M B S(Y)=s^{\prime} \mid D\right)$, where the terms are the MBS posterior of the set $s$ and the MBS posteriors for all its superset.

We calculate the interaction or redundancy [10] of a variable set $\left\{X_{i_{1}}, \ldots, X_{i_{n}}\right\}$ by computing $R=\frac{p\left(\left\{X_{i_{1}}, \ldots, X_{i_{n}}\right\} \mid D\right)}{\prod_{k=1}^{n} \underline{p}\left(\left\{X_{i_{k}}\right\} \mid D\right)}$.

Where the numerator is the a posteriori probability that the variable set is strongly relevant with respect to the target variable, and the denominator is the product of the a posteriori probability of the strong relevance of the variables in the set.

If $\mathrm{R}$ equals 1 , this means that the approximation of the strong relevance of the set under an independence assumption (i.e. the denominator) is equal to the real a posteriori probability of the set (i.e. the numerator). In this case, the variables in the set are independently relevant (i.e. they are independently present or absent in the Markov Blanket set of the target variable).

If $R$ differs from 1 , it indicates an interaction $(R>1)$ or a redundancy $(R<1)$. The corresponding Interaction Ratio is then $=\ln (R)$, and the Redundancy Ratio is $R R=-\ln (R)$.

\section{Results}

SNPs associated with ALL risk

As the blood sample was taken retrospectively and in remission, and only those samples were used where the normal leukocyte cell count was over $5 \mathrm{G} / \mathrm{l}$, practically no tumour cells were in our samples, thus we investigated only normal cells and germline polymorphisms.

From the 66 genotyped SNPs one SNP was monomorphic (minor allele frequency=0), and three had poor genotyping results (poor genotype clusters or low call rates), leaving 62 SNPs for frequentist and BNBMLA analyses.

Minor allele and genotype frequencies in controls and ALL patients are presented in Additional file 1. The results of the statistical analysis of all genotyping data are presented in Additional file 2.

The differences in allele and genotype frequencies between ALL cases and controls were nominally significant $(\mathrm{P}<0.05)$ for 20 SNPs. But, when gender adjusted logistic regression analysis with false discovery rate of (FDR $(\alpha)=) 1 \%$ significance threshold was calculated, the differences remained significant $(\mathrm{P}<3.42 \mathrm{E}-04)$ only in cases of 6 SNPs in two genes (rs10821936, rs7089424 and rs4506592 in $A R I D 5 B$, and rs6964969, rs11978267 and rs4132601 in IKZF1, as shown in Table 4). These results indicate that these SNPs are associated with increased susceptibility to ALL with odds ratios between 1.4 and 1.5. Then we analyzed whether the number of risk alleles

Table 3 Structural features that indicate different dependence types between the variables

\begin{tabular}{|c|c|c|}
\hline Relation & Abbreviation & Graphical \\
\hline \multicolumn{3}{|l|}{ Pairwise features } \\
\hline Direct causal relevance & $\operatorname{DCR}(X, Y)$ & There is an edge between $X$ and $Y$ \\
\hline Transitive causal relevance & $\operatorname{TCR}(X, Y)$ & There is directed path between $X$ and $Y$ \\
\hline Confounded relevance & $\operatorname{ConfR}(X, Y)$ & $X$ and $Y$ have common ancestor \\
\hline Association & $A(X, Y)$ & DCR or TCR or ConfR \\
\hline Pure interactionist relevance & $\operatorname{PIR}(X, Y)$ & $X$ and $Y$ have common child \\
\hline Strong relevance & $\operatorname{SR}(X, Y)$ & PIR or DCR \\
\hline \multicolumn{3}{|l|}{ Relevance of variable sets } \\
\hline Strong relevance & $\operatorname{MBS}(Y)$ & $\begin{array}{l}\text { The set consisting of Y's parents, its children, and the other parents } \\
\text { of its children (the Markov Blanket Set of } Y \text { ) }\end{array}$ \\
\hline \multicolumn{3}{|c|}{ Interaction models of relevant variables } \\
\hline Strong relevance & $M B G(Y)$ & $\begin{array}{l}\text { The subgraph that includes the nodes in the MBS and the incoming } \\
\text { edges into } Y \text { and into its children (the Markov Blanket Subgraph of } Y \text { ) }\end{array}$ \\
\hline
\end{tabular}



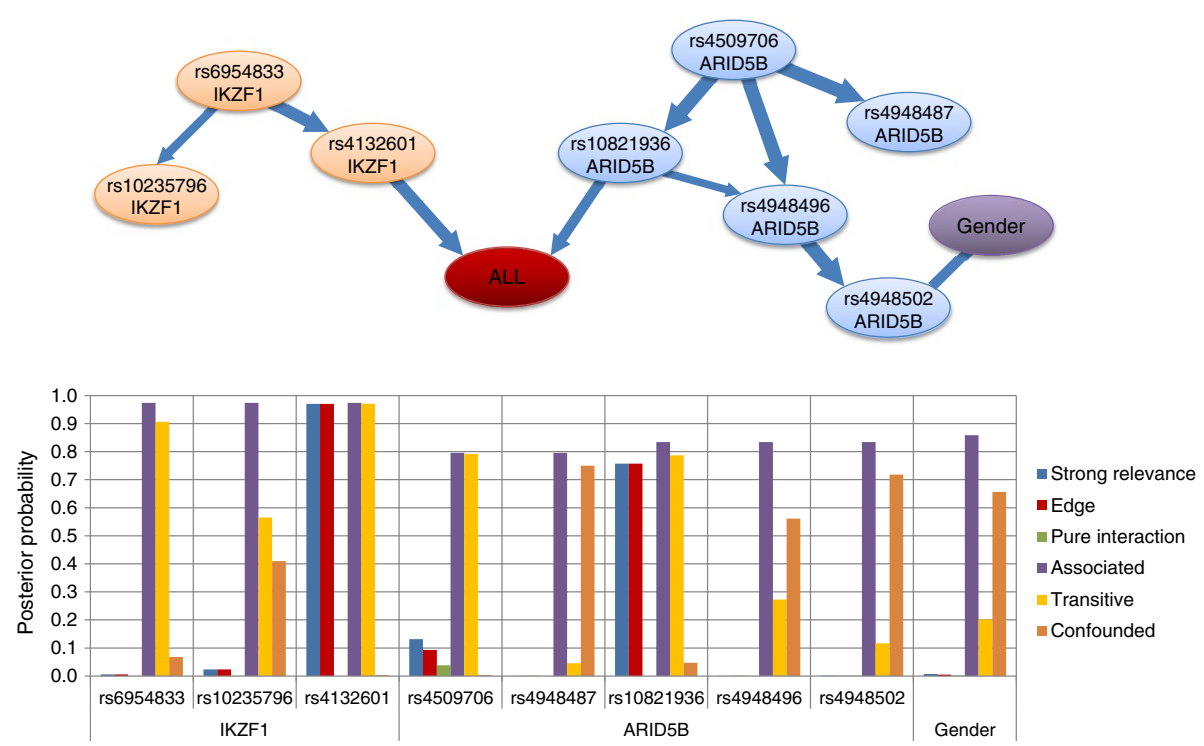

Figure 1 Illustration of different dependency relations between certain SNPs in ARID5B and IKZF1 genes, gender and ALL

susceptibility. Top panel: The "averaged structure" of the Bayesian networks including ALL susceptibility (red node), gender (purple node) and the SNPS of ARID5B (blue nodes) and IKZF1 (orange nodes). The width of the edges is proportional to their a posteriori probability. The probability of the edges is computed by averaging over the Bayesian networks visited by the MCMC process. See Methods. Bottom panel: The posterior probability of strong relevance (blue columns), edge (direct strong relevance, red columns), pure interaction (green columns), association (purple columns), transitive association (yellow columns) and confounded association (brown columns) of the variables to ALL susceptibility according to the BN-BMLA method.

of each SNP influenced the susceptibility to ALL, and found that in all cases the homozygous states are associated with higher risk (OR between 1.9 - 2.1) than the carrier status, or heterozygous states.

We calculated the linkage disequilibrium (LD) coefficients between the different SNPs (Additional file 3), and found that in both genes, the significantly associated SNPs were in strong linkage with each other. This means that there is only one, but strong signal in each gene. We also evaluated the effect of different haplotypes on the risk to ALL. We found two haplotypes (Additional file 4), which influenced the susceptibility to ALL in the IKZFI gene. But the odds ratio associated with the haplotypes, were not higher than in the case of individual SNPs. Interestingly, however, a haplotype containing the major alleles of the two individually associated SNPs (rs4132601, rs6964969) in the IKZF1 gene shows a slight protection against ALL (OR $=0.73$ (0.62-0.86); $\mathrm{P}=3.0 \mathrm{E}-04)$.

Subsequently, it was investigated whether these SNPs influence the clinical characteristics of ALL. As can be seen in Table 4 all the SNPs which were associated with ALL, increased the risk of B-cell ALL, but not of T-cell ALL. When the hyperdiploid ALL ( $\geq 50$ chromosomes) was considered, two SNPs in the STAT3 gene (rs3816769, rs12949918) showed decreased risk to this clinical subtype of the disease. As these SNPs are in LD

Table 4 Summary of the significant results of logistic regression analyses

\begin{tabular}{|c|c|c|c|c|c|c|c|c|c|c|c|c|c|}
\hline \multirow[t]{2}{*}{ Gene } & \multirow[t]{2}{*}{ SNP } & \multicolumn{3}{|c|}{ ALL } & \multicolumn{3}{|c|}{ B-ALL } & \multicolumn{3}{|c|}{ T-ALL } & \multicolumn{3}{|c|}{ HD-ALL* } \\
\hline & & P-value & OR & & P-value & OR & & & OR & 95\% C.I. & & OR & 95\% C.I. \\
\hline ZFY & rs6964969 & 1.67E-05 & 1.50 & $1.25-1.80$ & 1.17E-07 & 1.70 & $1.40-2.08$ & $1.88 \mathrm{E}-01$ & 0.76 & $0.50-1.14$ & $8.72 \mathrm{E}-02$ & 1.36 & 0.96-1.94 \\
\hline ZFI & rs11978267 & $2.46 \mathrm{E}-05$ & 1.50 & $1.24-1.79$ & 2.97E-07 & 1.68 & $1.38-2.05$ & $2.11 \mathrm{E}-01$ & 0.77 & $0.51-1.16$ & $5.08 \mathrm{E}-02$ & 1.42 & $1.00-2.0$ \\
\hline IKZFI & rs4132601 & $1.69 \mathrm{E}-05$ & 1.50 & $1.25-1.80$ & 2.22E-07 & 1.69 & $1.38-2.06$ & $3.46 \mathrm{E}-01$ & 0.83 & $0.55-1.23$ & 9.13E-02 & 1.36 & $0.95-1$. \\
\hline RID5B & rs10821936 & 7.31E-05 & 1.43 & $1.20-1.71$ & $1.95 \mathrm{E}-05$ & 1.53 & $1.26-1.85$ & $3.90 \mathrm{E}-01$ & 1.17 & $0.82-1.66$ & $5.72 \mathrm{E}-03$ & 1.61 & $115-27$ \\
\hline ARID5B & rs7089424 & 1.17E-04 & 1.42 & $1.19-1.69$ & $2.68 \mathrm{E}-05$ & 1.52 & & & 1.16 & & & 1.60 & $1.14-2.2$ \\
\hline ARID5B & rs4506592 & $1.72 \mathrm{E}-04$ & 1.40 & $1.18-1.67$ & $3.35 \mathrm{E}-05$ & 1.51 & $1.24-1.83$ & $6.70 \mathrm{E}-01$ & 1.08 & $0.76-1.54$ & $1.24 \mathrm{E}-02$ & 1.55 & 1 \\
\hline STAT3 & rs3816769 & $3.76 \mathrm{E}-02$ & 0.83 & $0.69-0.98$ & $4.23 \mathrm{E}-02$ & 0.82 & $0.67-0.99$ & 5.82E-01 & 0.91 & $0.64-1.29$ & $1.34 \mathrm{E}-04$ & 0.62 & $0.49-0.7$ \\
\hline STAT3 & rs12949918 & 5.15E-02 & 0.84 & $0.71-1.00$ & 6.37E-02 & 0.84 & 0.69-1.01 & 5.54E-01 & 0.90 & $0.64-1.27$ & 2.32E-04 & 0.64 & $0.50-0.8$ \\
\hline
\end{tabular}

*Hyperdiploid ALL; Tag SNPs are bold, as well as P-values, which reached the $p<3.42 E-04 ;$ FDR(a)=1\% significance threshold. 
with each other (Additional file 3), it also corresponds to one signal. Interestingly, these SNPs do not influence the risk to ALL in general.

We also investigated whether the gender of the patients influence the effect of the SNPs, but found no such effect.

\section{Survival of ALL patients}

We also investigated which factors influence the 5- yearsurvival of the patients. We have data about the survival rate in 516 cases (95\% of all patients). Our sample set contains similar rate of relapsed patients to what was observed in the whole population [8]. However, the rate of died patients is lower in our study population $(p<0.001)$. Patients who died during the chemotherapy due to therapy resistant progressive disease or due to infections or toxicities of therapy are underrepresented in our sample.

The overall survival rate was $85.5 \%(\mathrm{n}=441)$ in our childhood ALL population (Figure 2).

There was no significant difference between sex groups and study protocols in overall survivals. There was significant difference between risk groups in overall survivals $(\mathrm{p}=1 \mathrm{E}-7)$. The survival rates were $92.6 \%$ in the low, $87.0 \%$ in the medium and $62.3 \%$ in the high risk groups (Figure 3 ).

The event-free survival rate was $81.0 \%$. There was no significant difference between sex groups and study protocols in event-free survivals. There was significant difference between risk groups in event-free survivals $(\mathrm{p}=1 \mathrm{E}-7)$. The survival rates were $90.4 \%$ in the low, $82.6 \%$ in the medium and $60.4 \%$ in the high risk groups.

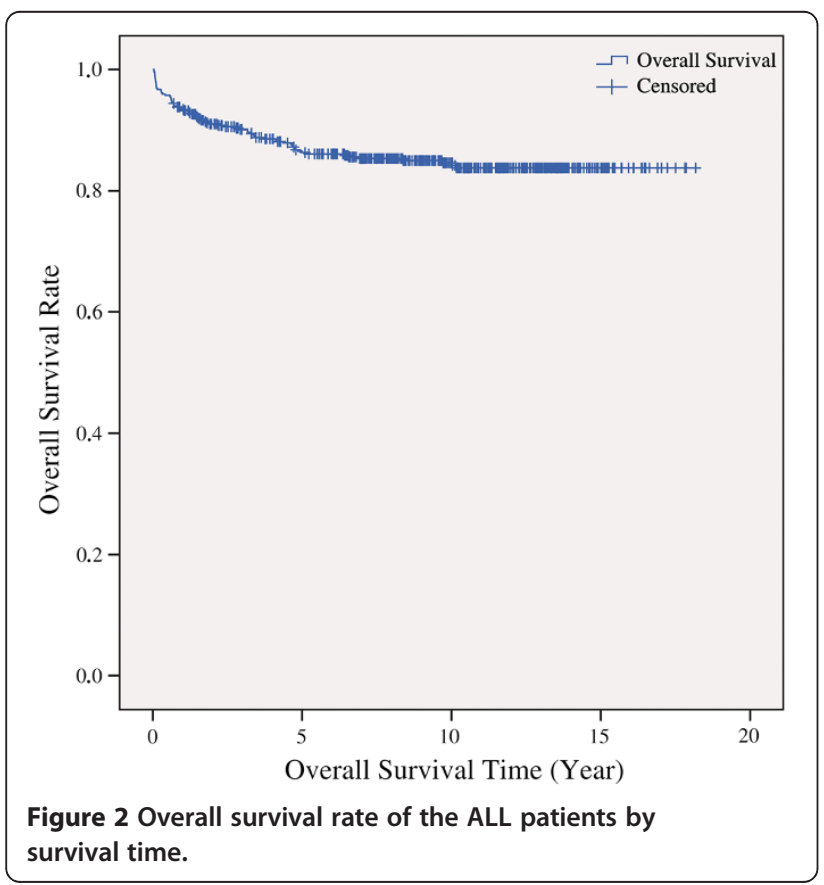

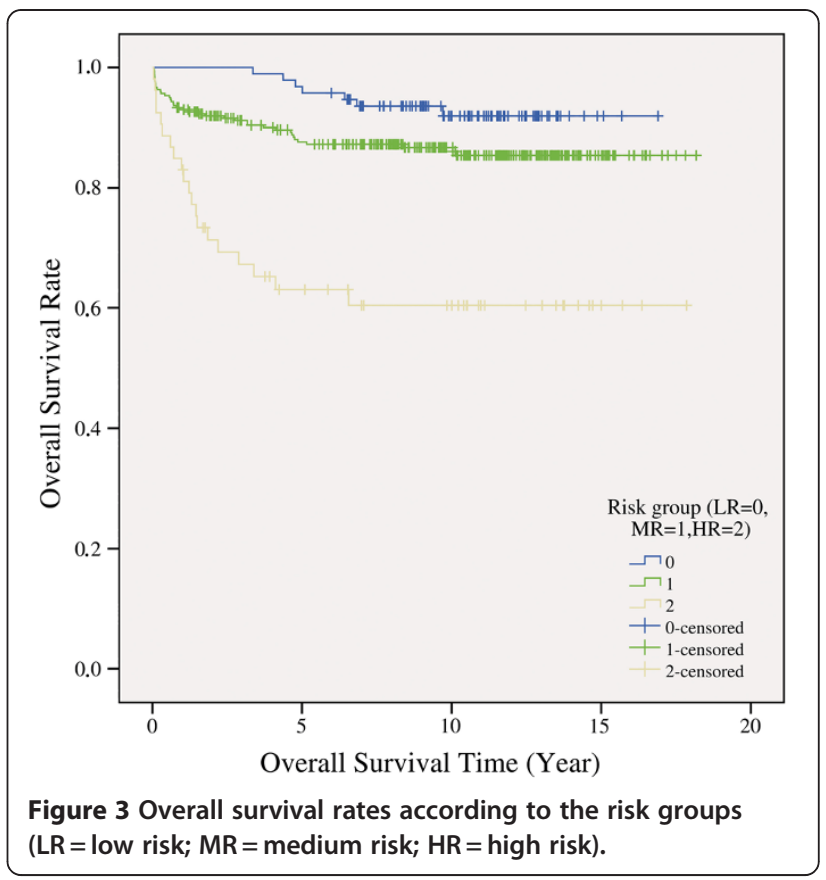

\section{SNPs influencing the survival of the ALL patients}

We investigated, whether the SNPs in our study influenced the survival of the patients. Altogether 4 SNPs in 3 genes (rs10403561 and rs874966 in CEBPA, rs3024979 in STAT6, rs11667351 in $B A X)$ were nominally significant in this respect, but none of them reached our significance threshold. Among these results the rs11667351 in the $B A X$ gene showed the strongest association and gave the lowest $\mathrm{p}$ value $(\mathrm{P}=0.001$; Additional file 5$)$. The detailed results of these SNPs can be seen in Additional file 6 . The overall and the event-free survival did not differ in this respect.

\section{BN-BMLA method for ALL susceptibility}

Besides evaluating our results with the traditional frequentist-based methods, we also analyzed them with our newly developed BN-BMLA method. Because the results and their interpretation differ from those of the standard statistical methods, here, in the results section we also give some explanations for their understandings.

As the results of the BN-BMLA are strongly influenced if the studied SNPs are in LD with each other, we selected 48 tag SNPs. Gender was also involved in the analysis as a variable. For each variable (SNPs and gender) we calculated posterior probability for strong relevance. Posterior values are between 0 and 1 , where 0 indicates no relevance, 1 indicates $100 \%$ relevance between a predictor and a target variable. According to our previous considerations [12,13], a variable is relevant when its posterior of strong relevance is greater than or equal to 0.5 . Above 0.75 the relevancy is regarded as convincing, between 0.5 and 0.75 as moderate. 


\begin{tabular}{|c|c|c|c|c|c|}
\hline Phenotype & ALL & B-ALL & T-ALL & HD-ALL & Risk group \\
\hline Gender & 0.01 & 0.06 & $0.85^{*}$ & 0.07 & 0.08 \\
\hline rs10821936 (ARID5B) & $0.76^{*}$ & $0.95^{*}$ & 0.02 & 0.14 & 0.05 \\
\hline rs12949918 (STAT3) & 0.01 & 0.00 & 0.01 & $0.60^{*}$ & 0.00 \\
\hline rs17405722 (STAT3) & 0.23 & 0.24 & 0.21 & 0.37 & 0.09 \\
\hline rs12457893 (BCL2) & 0.02 & 0.01 & 0.24 & $0.57^{*}$ & 0.05 \\
\hline rs1893806 (BCL2) & 0.02 & 0.00 & 0.02 & 0.01 & 0.42 \\
\hline rs3212713 (JAK3) & 0.03 & 0.11 & 0.17 & $0.56^{*}$ & 0.01 \\
\hline rs3087253(CCR5) & 0.03 & 0.10 & 0.15 & $0.56^{*}$ & 0.00 \\
\hline rs2282883 (AhR) & 0.01 & 0.00 & 0.11 & 0.08 & 0.42 \\
\hline rs2066853 (AhR) & 0.06 & 0.04 & 0.25 & 0.34 & 0.04 \\
\hline rs4132601 (IKZF1) & $0.97^{*}$ & $1.00^{*}$ & 0.02 & 0.02 & 0.00 \\
\hline
\end{tabular}

${ }^{*}$ relevant posteriors (Posterior probability > 0.5); HD-Hyperdiploid.

The results of the analysis of ALL risk are presented in Additional file 7. The most relevant SNPs and genes (i.e. with high posteriors for strong relevance) according to the BN-BMLA are presented in Table 5. In case of ALL susceptibility, the most relevant SNPs are rs10821936 in $A R I D 5 B$ and rs4132601 in IKZF1. The probability that these SNPs are directly associated to ALL is 0.76 for rs10821936 and 0.97 for rs4132601, respectively. Both of these direct associations are even more probable in case of the B-cell lineage sample group (0.95 and 1.0 for rs10821936 and rs4132601, respectively). However, in case of the T-cell lineage sample group, the probability of the direct relevance of these SNPs is very low, namely, around 0.02 . As the B-cell lineage is lot more frequent, the high probability of the strong relevance of these two SNPs in case of ALL susceptibility is probably due to their strong relevance in B-cell lineage.

In the T-cell lineage, only the gender of the patient has a high probability of direct association to ALL susceptibility (0.85), where males have significantly higher odds of developing ALL than women $(\mathrm{OR}=2.28$, C.I. 95\%: $1.32-3.93)$.

In the hyperdiploid sample group, the probabilities of the most relevant SNPs are moderate. Namely, rs12949918 in STAT3 has 0.6, rs12457893 in BCL2 has 0.57 , rs3212713 in JAK1 has 0.56, and rs3087253 in CCR5 has 0.56 probability of being strongly relevant to the hyperdiploid phenotype.

\section{Detailed characterization of association relations}

In the context of genetic association studies, strongly relevant variables with respect to a phenotype represent the genetic and phenotypic factors that directly influence the phenotype (e.g. disease susceptibility). However, the standard concept of pairwise association is not identical to the concept of strong relevance.
The association of a genetic variant to a phenotypic feature can have multiple types (Figure 1). First, the causal SNP has a direct influence on the phenotype. In this case the probability of the existence of a direct edge between the causal SNP and the phenotypic variable will be high. Second, due to linkage disequilibrium or evolutionary patterns, the genetic factors are often dependent on each other. Because of this dependency, univariate association tests can not necessarily make the distinction between the true causal SNP and the SNPs that are strongly linked to it. In these cases, the association is mediated by the causal SNP, and there exists a path between the associated SNP and the phenotypic variable with high probability. The association is transitive if the path is directed, and confounded if it is not. Third, if a SNP has no main effect on the phenotype, but has an epistatic effect along with another factor, then this SNP is not associated (according to the standard concept of association), but strongly relevant to the phenotype (i.e. it is in pure interaction with the phenotype).

We computed the a posteriori probability of the different association types with respect to ALL susceptibility in all sample groups (Additional file 7). Here, we present some examples how the results in Additional file 7 can be interpreted. The probability of the association of the SNPs in ARID5B (rs4509706, rs4948487, rs10821936, rs4948496, rs4948502) to ALL susceptibility are equal or greater than 0.8 , but only rs10821936 can be stated as directly relevant, because its posterior of strong relevance is 0.76 and the posterior of strong relevance is below 0.13 in case of all other SNPs, which indicates their non-causal, non-functional role. The situation is same in case of the SNPs in the IKZF1 gene. The association of the SNPs rs6954833, rs10235796, and rs4132601 in IKZF1 to ALL susceptibility is highly probable (0.97), but only rs 4132601 has a high probability $(0.97)$ of being strongly relevant. These effects are more expressed in the B-cell lineage sample group.

The probability of pure interaction is very low (below 0.1) in case of all SNPs in ALL susceptibility and B-cell lineage sample groups. However, in other sample groups, there are some SNPs with low probability of being in pure interaction to the phenotype, e.g. 0.43 in case of rs3212713 in JAK3 in the hyperdiploid sample group, and 0.42 in case of rs2282883 in $A H R$ when the target variable was the risk group of the patients.

\section{Redundancies and interactions}

The Bayesian analysis offers a principled way to compute the measure of interaction or redundancy of two (or more) SNPs. In this context, interaction means that two SNPs tend to occur together more often in the hypotheses of the strongly relevant variable sets than it is expected (i.e. the joint strong relevance of the two SNPs 

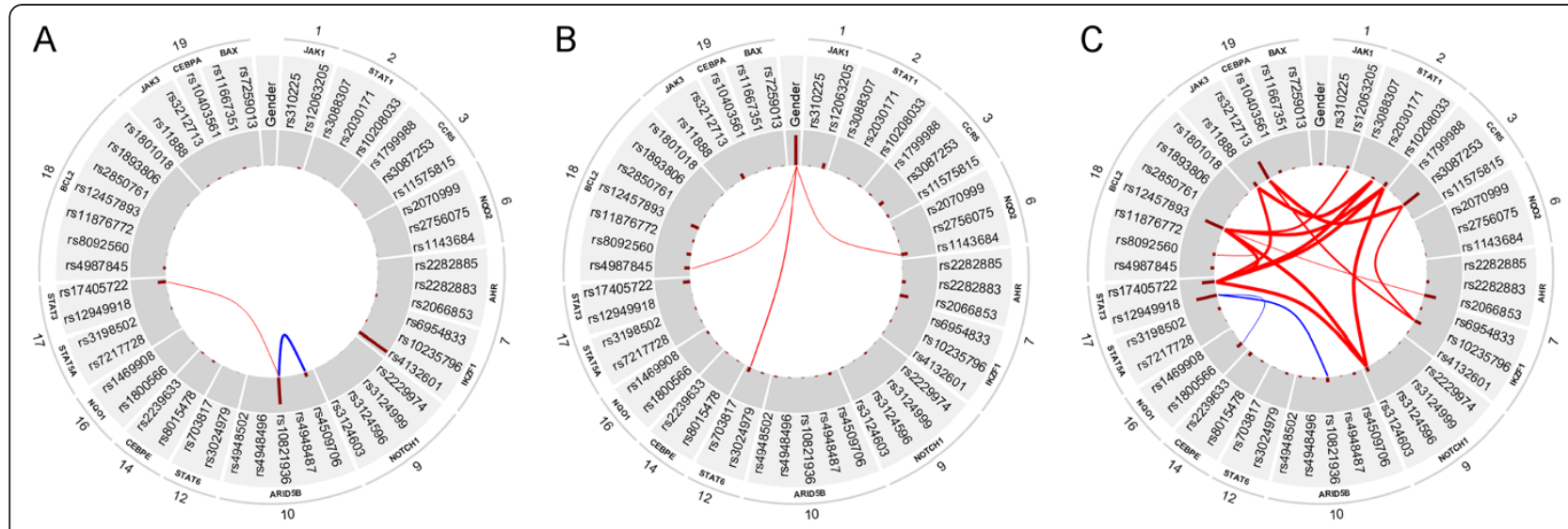

Figure 4 Redundancies and interactions according to the BN-BMLA method. The figure shows the magnitude of redundancies (blue curved lines) and interactions (red curved lines) between the variables in the whole dataset (i.e. ALL susceptibility, A panel), in the T-cell lineage sample group (B panel) and in the hyperdiploid sample group (C panel) according to the BN-BMLA method. See Methods for the computation of interaction and redundancy. The width of the curved lines is proportional to the strength of the effect. The a posteriori probability of the strong relevance of the variables is proportional to the length of the dark red columns next to the variable in the inner gray colored ring.

The corresponding genes and chromosomes of the SNPS are shown on the outer ring.

is more probable than it can be approximated from their univariate relevance). On the other hand, redundancy means that two SNPs are somewhat interchangeable in the hypotheses of the strongly relevant variable sets (i.e. the probability of the joint strong relevance of two SNPs is lower than it is expected). We computed the redundancies and interactions between all variables in case of all sample groups. In Figure 4, we present the results in a graphical form in case of the whole dataset (ALL susceptibility), the T-cell lineage patient group and the hyperdiploid patient group. The analysis did not result in any interactions or redundancies in case of the other sample groups.

In case of ALL susceptibility, rs10821936 in ARID5B and rs17405722 in STAT3 showed a weak interaction (Interaction ratio $(\mathrm{IR})=0.15$ ) and two SNPs in $A R I D 5 B$, namely rs10821936 and rs4509706 showed a moderate redundancy (Redundancy ratio $(R R)=0.33)$. $A R I D 5 B$ and IKZF1 (the two genes that have the highest posterior of strong relevance to ALL susceptibility) showed no interaction or redundancy with each other.

In case of T-cell lineage sample group, the gender showed a weak interaction with three SNPs in three genes, namely rs703817 in STAT6 $(\mathrm{IR}=0.16), \mathrm{rs} 4987845$ in $B C L 2$ (IR = 0.1), and rs1143684 in NQO2 ( $\mathrm{IR}=0.11)$. This latter could be confirmed by logistic regression analysis, as well. This indicated, that male status increased the risk of T cell ALL, but carrying an allele of rs1143684 slightly decreased the risk (interaction tag: $\mathrm{p}=0.039, \mathrm{OR}=0.565$, C.I. $95 \%: 0.33-0.97$ ).

In case of the hyperdiploid sample group, several overlapping components (i.e. sets of SNPs) were found that showed strong interaction. The component with the highest interaction ratio $(I R=3.06)$ consists of four SNPs in four genes, namely rs17405722 in STAT3, rs12457893 in BCL2, rs10208033 in STAT1, and rs3124603 in NOTCH1. The component with the second highest interaction ratio $(\mathrm{IR}=2.72)$ consists of three SNPs in three genes, namely rs17405722 in STAT3, rs11888 in JAK3, and rs2030171 in STAT1. The third component $(\mathrm{IR}=0.86)$ consists of three SNPs, rs3212713 in JAK3, rs3087253 in CCR5, and rs10235796 in IKZF1. Since these datasets are relatively small, exact characterization of these effects needs further validation.

\section{Survival analysis with BN BMLA}

All calculated posterior probabilities in event-free survival (EFS) and overall survival (OS) are presented in Additional file 8, and the most relevant variables in Table 6. In both cases, lineage (B- or T-cell) and risk group were found to be strongly relevant to the target variable (event-free and overall survival indicator) with high probability. However, it is more probable that lineage is in pure interaction $(\mathrm{P}=0.68$ in EFS) than it has a direct relevance $(P=0.09$ in EFS). Its effect is mediated by the risk group, thus lineage is important only if risk group is known (Figure 5).

In both cases, the strongly relevant genetic factors with the highest probability are rs11667351 in $B A X(0.79$ in EFS, and 0.87 in OS), and rs10403561 in CEBPA (0.63 in EFS, and 0.62 in OS). Besides, a SNP in STAT6, namely rs703817 can be indicated as strongly relevant in case of OS $(\mathrm{P}=0.67)$, but its probability is lower in case of EFS $(\mathrm{P}=0.34)$.

We computed the redundancies and interactions between all variables in both survival types, as well. The results are shown in Figure 6. 
Table 6 Posterior probabilities of the most relevant variables in event-free and overall survival

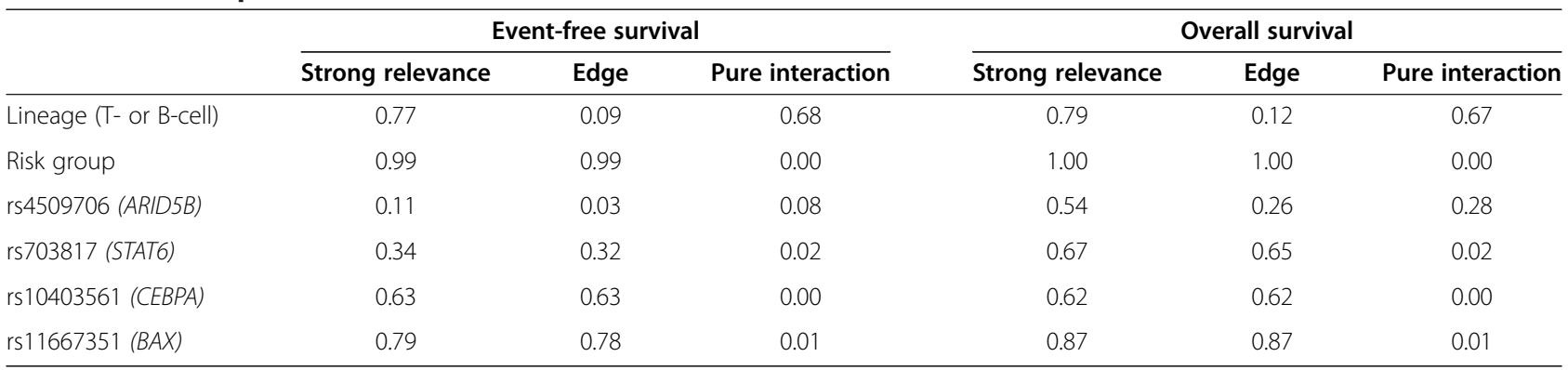

\section{Discussion}

In this study we presented our results of a candidate gene association study in childhood acute lymphoblastic leukemia evaluated by traditional frequentist-based methods and our newly developed BN-BMLA method.

According to the frequentist-based evaluation, among the successfully genotyped 62 SNPs in 19 genes, 6 SNPs in 2 genes associated with increased susceptibility to ALL. But, as the SNPs in both genes were in LD, this corresponded to one signal in each gene, namely in ARID5B and IKZF1. Both associations were specific for B-cell ALL, and in all loci the effect on risk was dose-dependent, as homozygous states associated with higher risks.

ARID5B belongs to a family of transcription factors important in embryonic development, cell type-specific gene expression, and cell growth regulation. SNPs in the gene have been found associated with ALL in several previous genome wide and candidate gene association studies and in different populations $[3,4,6,20]$. In some studies the associations were restricted to Bhyperdiploid ALL and to males. In our study no such difference was found between boys and girls, and the SNPs were not associated with hyperdiploidy, but the important role of the gene in B-cell ALL susceptibility was confirmed. It must be added, however, that all of the associated SNPs in the ARID5B gene are in intron, and presently it is not known, how they influence the risk to ALL.

SNPs in the IKZF1 gene were identified by independent genome wide association studies in Caucasian children; although later in some studies and in some populations the association was not confirmed [3,4,21]. IKZF1 (encoding the lymphoid transcription factor IKAROS) is deleted in approximately $80 \%$ of the Philadelphia chromosome-positive ALL with constitutively active BCR-ABL1 tyrosine kinase [22,23]. Furthermore, Ikaros proteins are master regulators of lymphocyte development, thus IKZF1 is a good candidate gene for ALL. Earlier, one of the SNPs, the rs4132601, which showed an association with increased susceptibility to ALL, and influenced in an in vitro system the expression level of the gene in a dose-dependent fashion, with lower expression being associated with the risk alleles [4]. Since human and mouse studies suggest that diminished expression of IKZF1 interrupts lymphocyte development, creating conditions that maintain the rapidly dividing lymphoblasts that characterize ALL, the lower

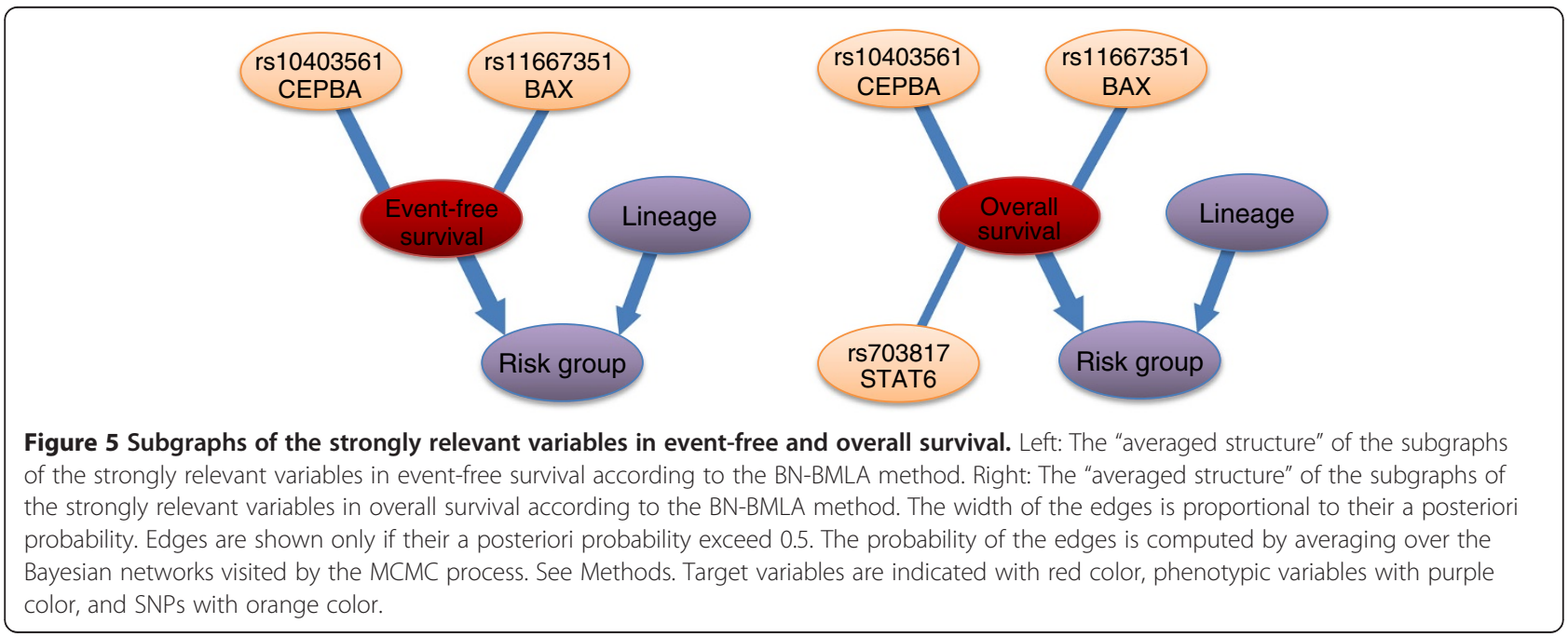



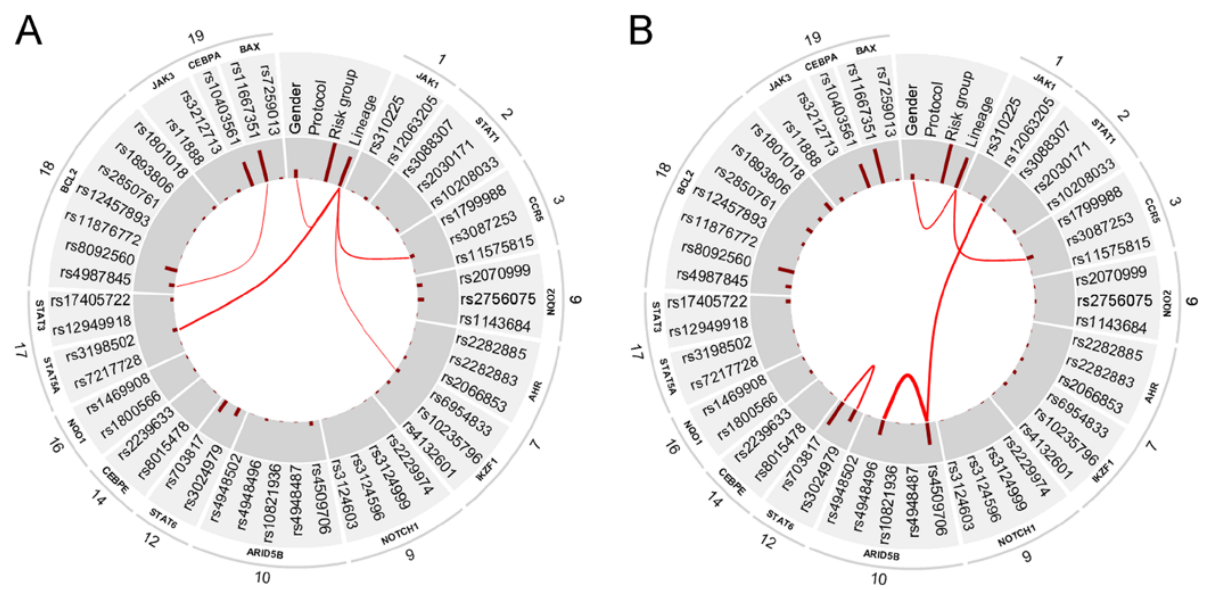

Figure 6 Interactions in event-free and overall survival according to the BN-BMLA method. The figure shows the magnitude of interactions (red curved lines) between the variables in the event-free survival (A panel), and in the overall survival (B panel) according to the BN-BMLA method. See Methods for the computation of interaction. The width of the curved lines is proportional to the strength of the effect. The a posteriori probability of the strong relevance of the variables is proportional to the length of the dark red columns next to the variable in the inner gray colored ring. The corresponding genes and chromosomes of the SNPs are shown on the outer ring.

expression associated with the rs4132601 might contribute to the increased risk to the disease.

SNPs in the STAT3 gene were found to decrease the risk to hyperdiploid ALL. The signal transducer and activator of transcription protein 3, encoded by STAT3, has been identified as a regulator of cell survival after exposure to apoptotic signals [24-26]. STAT3 serves, among others, as a substrate for SYK tyrosine kinase. SYK is capable of associating with and phosphorylating STAT3 in human B-lineage leukemia/lymphoma cells challenged with oxidative stress [27]. Inhibition of SYK with a small molecule drug candidate prevented oxidative stressinduced activation of STAT3 and overcame the resistance of human B-lineage leukemia/lymphoma cells to apoptosis. The decreased risk associated with the SNPs in the STAT3 gene in our study correlates with the results of other studies, e.g. the rs12949918 SNP was found to be associated with decreased susceptibility to different malignancies, like risk to B-cell non-Hodgkin lymphoma or renal cell carcinoma [28]. According to in vitro studies, the SNP affects STAT3 mRNA levels, with the minor allele having a lower STAT3 expression [29]. Presently it is not known how it might influence the risk to hyperdiploid ALL.

We also investigated whether the SNPs influence the survival of the disease. It must be noted, however, that the rate of died patients is lower in our study population $(\mathrm{p}<0.001)$, than in the whole Hungarian ALL population (see Methods), thus our results could be biased in this respect. In the frequentist-based evaluation, the rs11667351 in the $B A X$ gene showed the strongest association and gave the lowest $\mathrm{p}$ value $(\mathrm{P}=1 \mathrm{E}-3)$, but it still did not reach the significance threshold $(\mathrm{P} \leq 3.42 \mathrm{E}-4)$.
Next, we evaluated our results with the BN-BMLA method. Earlier, we applied this method to evaluating a partial genome screening in asthma [13]. In that study the frequentist-based method identified two genes for asthma susceptibility (FRMD6 and PTGDR), while the BN-BMLA identified 3 additional genes. When we analyzed the cause of this difference, it turned out that the other 3 genes indirectly associated with asthma risk, i.e. in different gene-gene interactions. Then, as BN-BMLA is also capable of analyzing multiple targets, we involved additional phenotypic, target variables in the analysis. In this case the BN-BMLA identified 3 additional genes. The SNPs in these genes influenced the susceptibility to asthma through other target variables, like rhinitis, IgE, or eosinophil levels. As all of these phenotypic variables are in strong association with asthma, association with these might also cause association with the disease. This latter is called transitive associations.

In the present study we could not involve additional target variables in the evaluation, as there are no common known phenotypic characteristics in controls, which significantly change the risk to ALL. The BNBMLA could confirm the association of SNPs in the $A R I D 5 B$ and IKZF1 to B-cell ALL with high posterior probability. Additionally, however, as explained in the detailed characterization of association relation, the results of the analysis gave additional information about the nature of the relations between the SNPs and the disease. In this case no strongly relevant interactions were found, but the analysis suggested several weak interactions. E.g. in case of ALL susceptibility, rs10821936 in ARID5B and rs17405722 in STAT3 showed a weak interaction, and in case of T-cell lineage 
sample group, the gender showed a weak interaction with three SNPs in three genes (Figure 4). Interestingly e.g., as it is also known from the scientific literature, the male gender increased the risk of T-cell ALL, but carrying an allele of rs1143684 in the NQO2 gene slightly decreased the risk. The BN-BMLA is also capable of calculating redundancy of the variables. E.g. in the present study the rs10821936 and rs4509706 SNPs in ARID5B gene, showed a moderate redundancy, i.e. their effects are interchangeable with each other. It is also an important finding, that there are no interactions or redundancies between the two most relevant genes, ARID5B and $I K Z F 1$. This is also a confirmation of the results of other studies where no interactions were found between the two genes [30].

Several interactions have been detected in hyperdiploid ALL (Figure $4 \mathrm{C}$ ). Although the number of patients in this group is relative low, thus the results must be handled with some reservations, most of these interactions are biologically plausible. E.g. in our analysis a strong interaction was found among SNPs in the NOTCH1, STAT1, STAT3 and BCL2 genes. In the scientific literature it is known that STAT3 is activated in the presence of active Notch. Notch-IC stable transfectants increased STAT1-dependent transcription in response to IFN-gamma. In a zebra fish model of human NOTCH1induced T-cell leukemia, the leukemia onset was dramatically accelerated when the transgenic fish was crossed with another line over expressing the zebra fish $B c l 2$ gene, indicating synergy between the Notch pathway and the $B c l 2$-mediated antiapoptotic pathway [31-33]. All of these results suggest that the pathways represented by these genes are overlapping and there are interactions between them.

Analyzing the effects of the variables on the survival of the patients with BN-BMLA resulted in two SNPs in two genes with strong relevance. The relevance of the rs11667351 SNP in the $B A X$ gene was convincing (posterior probability $>0.75$ ), while that of the rs 1040356 in the CEBPA gene was moderate. As can be seen in Figure 6, the SNP in the $B A X$ gene was also involved in an interaction with a SNP in the BCL2 gene. This interaction is biologically plausible, as there are a lot of known interactions between the products of the two genes [34]. E.g. BCL2 prevents BAX/BAK oligomerization, and BCL2 binds to and inactivates BAX. There are also data, although inconclusive, about the role of BAX in the relapse of children with ALL. High levels of BAX protein have been associated with an increased probability of relapse in one study, while in another study both BAX expression levels and the BAX/BCL2 ratio were significantly lower in samples at relapse compared to samples at initial diagnosis [35,36]. In our study, children homozygous to the minor allele of the rs11667351 SNP had a very poor survival rate $(40 \%$, Additional file 5 and Additional file 6). In a study, this variant was associated with lower $B A X$ mRNA in lymphocytes. It must be added, however, that the number of patients homozygous to the minor allele of the rs11667351 was very low in our population.

The protein encoded by CEBPA is a transcription factor which can bind as a homodimer to certain promoters and enhancers. Its mutations have been found to be implicated in acute myelogenous leukemia with favorable prognosis [37]. In our study minor allele carrier status showed better survival rate, than major allele homozygotes, suggesting certain concordance between the two observations.

The BN-BMLA also detected known connections between risk groups, lineage and survival, but also revealed some possible interactions between certain variables like gene-gene, lineage-gene or lineage-gender (Figure 6).

Evaluating the effect of SNPs on the survival rate of the patients resulted in some discrepancies between the two methods. The frequentist-based method detected only nominally significant associations, which, according to the accepted rules, have to be rejected, while the BN-BMLA, especially in the case of the $B A X$ gene found strong, convincing relevancy. It is generally accepted, that the frequentist-based methods cannot properly handle the multiple testing problem. To avoid type I error, sometimes the frequentist methods are too conservative and unable to detect weak effects or interactions. The findings of the BN-BMLA are biologically plausible, but additional studies are needed to confirm these results.

In the present paper we show the ability of BN-BMLA to evaluate a candidate gene association study. As can be seen from the results, the advantage is not that the BNBMLA can detect more relevant variables, but the Bayesian networks offer a rich language for the detailed representation of types of relevance, including direct and indirect aspects. Additionally, Bayesian statistics offer an automated and normative solution for the multiple hypothesis testing problems [11]. In this study we could not utilize the full potential of the BN-BMLA, since we could not include multiple targets present also in controls, we did not have data from different sources (e.g. from gene expression analysis) and did not involve data from other databases and did not involve a priori knowledge in our evaluation. Furthermore, the investigated population was relative small. Childhood acute lymphoblastic leukemia is a relative rare disease, with an incidence of 50-70 cases in a year in Hungary. In this respect the 543 ALL children in this study can be regarded as a large population. However, in a gene 
association study, where 62 SNPs are investigated, it is very difficult to detect in such a population weak effects or gene-gene interactions. Still, the BN-BMLA was able to reveal, besides the strongly relevant $A R I D 5 B$ and $I K Z F 1$ polymorphisms, several possible interactions, and showed the possible types of them. According to our studies in larger, artificial datasets, if there are real complex interactions among the variables, the method is able to reveal complex network of interactions, significantly more complex than in Figure 1 in this study [12].

The Bayesian statistical framework allows the calculations of posteriors over a wide range of hypotheses, such as strong relevance of variables, pairs of variables, triplets of variables, etc. [9-13]. This shows the advantage of the Bayesian framework, because it allows the selection of appropriate level of complexity of hypotheses, which is not possible in the traditional hypothesis testing approach. Furthermore, this Bayesian global relevance analysis method provides posteriors, which are direct statements about hypotheses, thus it can also be used to construct probabilistic data analytic knowledge bases in genetic association studies to support complex quering, off-line meta-analysis, and fusion with background knowledge.

We offer the BN-BMLA method for academic purposes. The tool is available at a public website [38].

\section{Conclusion}

In the present study we confirmed the role of genetic variations in $A R I D 5 B$ and IKZF1 in the susceptibility to $B$-cell ALL. In our population we found that genetic variations in the STAT3 gene might influence the susceptibility to hyperdiploid ALL. We presented the survival rate of a relatively large number of Hungarian children with ALL, and investigated the effects of some genetic and other variables on the survival rate. We also evaluated our results with our newly developed BN-BMLA method, which confirmed the relevance of SNPs in the $A R I D 5 B$ and IKZF1 genes in B-cell ALL with high posterior probabilities. Furthermore, the results of the analysis gave additional information about the nature of the relations between several SNPs and the disease. In the different subgroups of patients, the BN-BMLA revealed several types of relations. Evaluating the survival rates of the patients, the BN-BMLA showed that besides risk groups and subtypes, genetic variations in the $B A X$ and $C E B P A$ genes might also influence the survival of the patients.

In the present paper we showed several advantageous features of the BN-BMLA method, and we demonstrated that in gene association studies it might be a useful supplementary to the traditional frequentist-based statistical method.

\section{Additional files} Additional file 1: Minor allele and genotype frequencies (\%) in
pediatric ALL ( $n=543)$ and control $(n=529)$ patients.

Additional file 2: Results of the statistical analysis of all genotyping data.

Additional file 3: Linkage disequilibrium between some SNP pairs.

Additional file 4: Haplotypes in the IKZF1 gene, which significantly influenced the risk of ALL.

Additional file 5: Survival rates in the different genotype groups according to rs11667351 in the BAX gene.

Additional file 6: Results of the statistical evaluations of the influence of some SNPs on the overall survival time.

Additional file 7: The a posteriori probabilities of the different association types in ALL susceptibility.

Additional file 8: The a posteriori probabilities of the different association types in event-free and overall survival.

\section{Abbreviations}

ALL: Acute lymphoblastic leukemia; BN-BMLA: Bayesian network based Bayesian multilevel analysis of relevance; DAG: Directed acyclic graph; EFS: Event-free survival; OS: Overall survival; HWE: Hardy-Weinberg Equilibrium; LD: Linkage Disequilibrium; BFM: Berlin-Frankfurt-Münster protocol; SNP: Single nucleotide polymorphism; FDR: False discovery rate; $\mathrm{MC}^{3}$ : Metropolis Coupled Markov Chain Monte Carlo method.

\section{Competing interests}

The authors of this paper declare that they have no competing interests.

\section{Authors' contributions}

OLC: Conceived and designed the experiments, participated in the drafting of the manuscript, evaluated the results; AG: Designed the software used in analysis, carried out Bayesian statistical evaluation, participated in the drafting of the manuscript; ÁFS: participated in the drafting of the manuscript, conceived and designed the experiments; DJE: participated in the drafting of the manuscript, was responsible for clinical data; PA: designed the software used in analysis, carried out statistical evaluation; GS: carried out the molecular genetic studies, evaluated the results; NK: carried out the molecular genetic studies; KC: was responsible for clinical data; $\mathrm{MH}$ : evaluated the results, was responsible for the clinical data; GK: was responsible for the clinical data; AF: participated in the drafting of the manuscript; CS: organized the study, participated in the design of the study and performed the statistical analysis, participated in the drafting of the manuscript. All authors read and approved the final manuscript.

\section{Acknowledgements}

This study was supported by OTKA (Hungarian Scientific Research Fund): K81941 (C. Szalai); TS/2 044707 (A. Falus); and the Economic Competitiveness Operational Program, Hungary GVOP 3.1.1-2004-05-0022/3.0 (A Falus, DJ Erdélyi); OTKAPD (Hungarian Scientific Research Fund):76348 (P.Antal), Bolyai Grant (Hungarian Academy of Science): (P.Antal) and NKTH (National Research and Technology) TECH_08-A1/2-2008-0120: (A. Falus, C. Szalai, P. Antal).

This work is connected to the scientific program of the " Development of quality-oriented and harmonized $\mathrm{R}+\mathrm{D}+\mathrm{I}$ strategy and functional model at BME" project. This project is supported by the New Széchenyi Plan (Project ID: TÁMOP-4.2.1/B-09/1/KMR-2010-0002).

Web pages for the funding organizations:

OTKA: http://www.otka.hu/

NKTH: www.nih.gov.hu

ETT: http://www.ett.hu/palyazat.htm

Bolyai Grant: http://www.bolyaitestamentum.hu/?m=24

\section{Author details}

${ }^{1}$ Department of Genetics, Cell- and Immunobiology, Semmelweis University, Budapest, Nagyvárad tér 4 H-1089, Hungary. ${ }^{2}$ Department of Measurement and Information Systems, University of Technology and Economics, Budapest, Hungary. ${ }^{3}$ 2nd Department of Pediatrics, Semmelweis University, 
Budapest, Hungary. ${ }^{4}$ Heim Pal Children Hospital, Budapest, Hungary. ${ }^{5}$ Csertex Research Laboratory, Budapest, Hungary.

Received: 27 March 2012 Accepted: 21 September 2012 Published: 28 September 2012

\section{References}

1. Sherborne AL, Hemminki K, Kumar R, Bartram CR, Stanulla M, Schrappe M, Petridou E, Semsei AF, Szalai C, Sinnett D, et al: Rationale for an international consortium to study inherited genetic susceptibility to childhood acute lymphoblastic leukemia. Haematologica 2011, 96(7):1049-1054

2. Sherborne AL, Hosking FJ, Prasad RB, Kumar R, Koehler R, Vijayakrishnan J, Papaemmanuil E, Bartram CR, Stanulla M, Schrappe M, et al: Variation in CDKN2A at 9p21.3 influences childhood acute lymphoblastic leukemia risk. Nat Genet 2010, 42(6):492-494.

3. Treviño LR, Yang W, French D, Hunger SP, Carroll WL, Devidas M, Willman C, Neale G, Downing J, Raimondi SC, et al: Germline genomic variants associated with childhood acute lymphoblastic leukemia. Nat Genet 2009, 41(9):1001-1005.

4. Papaemmanuil E, Hosking FJ, Vijayakrishnan J, Price A, Olver B, Sheridan E, Kinsey SE, Lightfoot $T$, Roman E, Irving JAE, et al: Loci on 7p12.2, 10q21.2 and $14 q 11.2$ are associated with risk of childhood acute lymphoblastic leukemia. Nat Genet 2009, 41(9):1006-1010.

5. Semsei AF, Erdélyi DJ, Ungvári I, Kámory E, Csókay B, Andrikovics H, Tordai A, Cságoly E, Falus A, Kovács GT, et al: Association of some rare haplotypes and genotype combinations in the MDR1 gene with childhood acute lymphoblastic leukaemia. Leuk Res 2008, 32(8):1214-1220.

6. Healy J, Richer C, Bourgey M, Kritikou EA, Sinnett D: Replication analysis confirms the association of ARID5B with childhood B-cell acute lymphoblastic leukemia. Haematologica 2010, 95(9):1608-1611.

7. Moore JH, Asselbergs FW, Williams SM: Bioinformatics challenges for genome-wide association studies. Bioinformatics 2010, 26(4):445-455

8. Semsei AF, Antal P, Szalai C: Strengths and weaknesses of gene association studies in childhood acute lymphoblastic leukemia. Leuk Res 2010, 34(3):269-271.

9. Antal P, Hullam G, Gezsi A, Millinghoffer A: Learning complex bayesian network features for classification. In Proceeding of third European Workshop on Probabilistic Graphical Models: 2006. Prague: Czech Republic; 2006:9-16.

10. Antal P, Millinghoffer A, Hullam G, Szalai C, Falus A: A Bayesian view of challenges in feature selection: feature aggregation, multiple targets, redundancy and interaction. In JMLR Workshop and Conference Proceedings: New challenges for feature selection in data mining and knowledge discovery: 2008. Belgium: Antwerpen; 2008:74-89.

11. Antal P, Millinghoffer A, Hullám G, Hajós G, Szalai C, Falus A: A bioinformatic platform for a Bayesian, multiphased, multilevel analysis in immunogenomics. In Bioinformatics for Immunomics, Immunomics reviews. 3rd edition. Edited by Flower D, Davies M, Ranganathan S. New York, USA: Springer; 2010:157-185.

12. Hullam G, Antal P, Szalai C, Falus A: Evaluation of a Bayesian model-based approach in GA studies. In Proceedings of the third International Workshop on Machine Learning in Systems Biology: 2010. Slovenia: Ljubljana; 2010:30-43.

13. Ungvári I, Hullám G, Antal P, Kiszel P, Gézsi A, Hadadi É, Virág V, Hajós G, Millinghoffer A, Nagy A, et al: Evaluation of a partial genome screening of two asthma susceptibility regions using Bayesian network based Bayesian multilevel analysis of relevance. PLoS One 2012, 7(3):e33573.

14. International HapMap Project-HapMap Database. http://www.hapmap.org.

15. Tests for deviation from Hardy-Weinberg equilibrium and tests for association. http://ihg2.helmholtz-muenchen.de/cgi-bin/hw/hwa1.pl.

16. Cooper GF, Herskovits E: A Bayesian method for the induction of probabilistic networks from data. Mach Learn 1992, 9(4):309-347.

17. Altekar G, Dwarkadas S, Huelsenbeck JP, Ronquist F: Parallel Metropolis coupled Markov chain Monte Carlo for Bayesian phylogenetic inference. Bioinformatics 2004, 20(3):407-415.

18. Gelman A, Carlin JB, Stern HS, Rubin DB, Dunson DB: Bayesian Data Analysis. 3rd edition. London: Chapman \& Hall; 1995

19. Gamerman D: Markov Chain Monte Carlo. London: Chapman and Hall; 1997.

20. Lahoud MH, Ristevski S, Venter DJ, Jermiin LS, Bertoncello I, Zavarsek S, Hasthorpe S, Drago J, de Kretser D, Hertzog PJ, et al: Gene targeting of
Desrt, a novel ARID class DNA-binding protein, causes growth retardation and abnormal development of reproductive organs. Genome Res 2001, 11(8):1327-1334.

21. Han S, Lee KM, Park SK, Lee JE, Ahn HS, Shin HY, Kang HJ, Koo HH, Seo JJ, Choi JE, et al: Genome-wide association study of childhood acute lymphoblastic leukemia in Korea. Leuk Res 2010, 34(10):1271-1274.

22. Molnár A, Wu P, Largespada DA, Vortkamp A, Scherer S, Copeland NG Jenkins NA, Bruns G, Georgopoulos $K$ : The lkaros gene encodes a family of lymphocyte-restricted zinc finger DNA binding proteins, highly conserved in human and mouse. J Immunol 1996, 156(2):585-592.

23. Mullighan CG, Miller CB, Radtke I, Phillips LA, Dalton J, Ma J, White D, Hughes TP, Le Beau MM, Pui CH, et al: BCR-ABL1 lymphoblastic leukaemia is characterized by the deletion of Ikaros. Nature 2008, 453(7191):110-114.

24. Otero DC, Poli V, David M, Rickert RC: Cutting edge: inherent and acquired resistance to radiation-induced apoptosis in B cells: a pivotal role for STAT3. J Immunol 2006, 177(10):6593-6597.

25. Haga S, Terui K, Zhang HQ, Enosawa S, Ogawa W, Inoue H, Okuyama T, Takeda K, Akira S, Ogino T, et al: Stat3 protects against Fas-induced liver injury by redox-dependent and -independent mechanisms. J Clin Invest 2003, 112(7):989-998.

26. Grandis JR, Drenning SD, Zeng Q, Watkins SC, Melhem MF, Endo S, Johnson DE, Huang L, He Y, Kim JD: Constitutive activation of Stat3 signaling abrogates apoptosis in squamous cell carcinogenesis in vivo. Proc Natl Acad Sci U S A 2000, 97(8):4227-4232.

27. Uckun FM, Qazi S, Ma H, Tuel-Ahlgren L, Ozer Z: STAT3 is a substrate of SYK tyrosine kinase in B-lineage leukemia/lymphoma cells exposed to oxidative stress. Proc Natl Acad Sci U S A 2010, 107(7):2902-2907.

28. Butterbach $K$, Beckmann L, de Sanjose S, Benavente Y, Becker N, Foretova L, Maynadie M, Cocco P, Staines A, Boffetta P, et al: Association of JAK-STAT pathway related genes with lymphoma risk: results of a European casecontrol study (EpiLymph). Br J Haematol 2011, 153(3):318-333.

29. Ito N, Eto M, Nakamura E, Takahashi A, Tsukamoto T, Toma H, Nakazawa H, Hirao $Y$, Uemura $H$, Kagawa $S$, et al: STAT3 polymorphism predicts interferon-alfa response in patients with metastatic renal cell carcinoma. J Clin Oncol 2007. 25(19):2785-2791.

30. Prasad RB, Hosking FJ, Vijayakrishnan J, Papaemmanuil E, Koehler R, Greaves M, Sheridan E, Gast A, Kinsey SE, Lightfoot T, et al: Verification of the susceptibility loci on 7p12.2, 10q21.2, and 14q11.2 in precursor B-cell acute lymphoblastic leukemia of childhood. Blood 2010, 115(9):1765-1767.

31. Gu F, Ma Y, Zhang Z, Zhao J, Kobayashi H, Zhang L, Fu L: Expression of Stat 3 and Notch 1 is associated with cisplatin resistance in head and neck squamous cell carcinoma. Oncol Rep 2010, 23(3):671-676.

32. Kamakura S, Oishi K, Yoshimatsu T, Nakafuku M, Masuyama N, Gotoh Y: Hes binding to STAT3 mediates crosstalk between Notch and JAK-STAT signalling. Nat Cell Biol 2004, 6(6):547-554.

33. Chen J, Jette C, Kanki JP, Aster JC, Look AT, Griffin JD: NOTCH1-induced Tcell leukemia in transgenic zebrafish. Leukemia 2007, 21(3):462-471.

34. Tzifi F, Economopoulou C, Gourgiotis D, Ardavanis A, Papageorgiou S, Scorilas A: The role of BCL2 family of apoptosis regulator proteins in acute and chronic leukemias. Adv Hematol 2012, 2012:524308.

35. Hogarth LA, Hall AG: Increased BAX expression is associated with an increased risk of relapse in childhood acute lymphocytic leukemia. Blood 1999, 93(8):2671-2678.

36. Prokop A, Wieder T, Sturm I, Essmann F, Seeger K, Wuchter C, Ludwig WD, Henze G, Dorken B, Daniel PT: Relapse in childhood acute lymphoblastic leukemia is associated with a decrease of the $\mathrm{Bax} / \mathrm{BCl}-2$ ratio and loss of spontaneous caspase-3 processing in vivo. Leukemia 2000, 14(9):1606-1613.

37. Preudhomme C, Sagot C, Boissel N, Cayuela JM, Tigaud I, de Botton S, Thomas X, Raffoux E, Lamandin C, Castaigne S, et al: Favorable prognostic significance of CEBPA mutations in patients with de novo acute myeloid leukemia: a study from the Acute Leukemia French Association (ALFA). Blood 2002, 100(8):2717-2723.

38. Genagrid Homepage. http://redmine.genagrid.eu/projects/ bayeseyedownload/wiki.

doi:10.1186/1755-8794-5-42

Cite this article as: Lautner-Csorba et al: Candidate gene association study in pediatric acute lymphoblastic leukemia evaluated by Bayesian network based Bayesian multilevel analysis of relevance. BMC Medical Genomics 2012 5:42. 